HNO 2022 $\cdot 70: 287-294$

https://doi.org/10.1007/s00106-021-01107-z

Angenommen: 3 . August 2021

Online publiziert: 20. September 2021

(c) Springer Medizin Verlag $\mathrm{GmbH}$, ein Teil von Springer Nature 2021

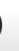

\section{Zur Effektivität der digitalen Vermittlung praktischer Fertigkeiten in der curricularen HNO-Lehre}

\author{
F. Krauss ${ }^{1} \cdot$ M. Giesler ${ }^{2} \cdot$ C. Offergeld ${ }^{1}$ \\ ${ }^{1}$ Klinik für Hals-Nasen-Ohrenheilkunde, Medizinische Fakultät, Universitätsklinikum Freiburg, Albert- \\ Ludwigs-Universität Freiburg, Freiburg, Deutschland \\ ${ }^{2}$ Freiburg, Deutschland
}

\section{Zusammenfassung}

Hintergrund: Aufgrund der aktuellen COVID-19-Pandemie musste innerhalb kurzer Zeit das curriculare Lehrangebot an medizinischen Fakultäten digitalisiert werden. Im Hinblick auf die Vermittlung praktischer Fertigkeiten stellt dies eine besondere Herausforderung dar.

Zielsetzung: Überprüft wurde, inwieweit praktische Fertigkeiten im Rahmen eines komplett digital umgesetzten HNO-Spiegelkurses erlernt werden können. Dabei wurde die Mindestmenge der zur sicheren Ausführung benötigten Unterrichtseinheiten (UE) identifiziert und der Schweregrad der Untersuchungen bestimmt.

Methodik: Während eines fünftägigen HNO-Spiegelkurses erlernten insgesamt 146 Studierende in täglich stattfindenden Videokonferenzen 6 HNO-Spiegeluntersuchungen. Eine Teilgruppe $(n=48)$ wurde an allen Tagen formativ geprüft und der Kompetenzzuwachs über die fünf Tage statistisch analysiert. Am fünften Tag wurden Prüfungen mit der gesamten Kohorte durchgeführt.

Ergebnisse: Im Verlauf der fünf Tage wurde für die Teiluntersuchungen jeweils ein signifikanter Leistungszuwachs festgestellt. Hierbei konnten Untersuchungen als "leicht" und "schwer" identifiziert werden. Eine Leistungssättigung, entsprechend der Kompetenzstufe 3a des Nationalen Kompetenzbasierten Lernzielkatalogs Medizin (NKLM), wurde an Tag 4 nach 5 UE erreicht.

Schlussfolgerung: Praktische Fertigkeiten können auch im Rahmen von digital durchgeführten Lehrveranstaltungen erworben werden. Einschränkungen ergeben sich bei der Qualität der Untersuchungsmaterialien und bei der Befunderhebung. Darüber hinaus kann durch eine geeignete Gewichtung des Untersuchungsfokus eine ökonomische Curriculumplanung gelingen.

\section{Schlüsselwörter}

COVID-19 · Digitalisierung · Lernerfolgsmessung · Blended Learning · Medizinstudierende

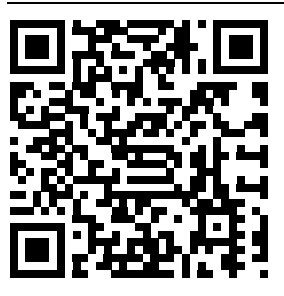

QR-Code scannen \& Beitrag online lesen
„Präsenzlehre ist unersetzlich!“ [19]. Das ist die zentrale Botschaft der gleichnamigen Senatsresolution der Universität Freiburg vom 31. Juli 2020 zur Lehr- und Lernsituation im Sommersemester 2020, das ganz im Zeichen der COVID-19-Pandemie stand. Es bleibt die Frage, ob die hier als „eine Ausnahmelösung in einer Ausnahmesituation" beschriebene Momentaufnahme nicht vielmehr den Einstieg in das Lehrkapitel der Zukunft skizziert. Dieser Einstieg begann mit der notgedrungen kompletten Umstellung auf Online-Lehre, was die deutschen Universitäten z. T. vor Probleme in der digitalen Realisierung eines tragfähigen Konzepts stellte [14].

In der curricularen Lehre der medizinischen Fakultäten hat die digitale Transformation schon präpandemisch Einzug gehalten, jedoch mit Unterschieden in Ausmaß und Qualität [16]. Studien der vergangenen Jahre konnten aufzeigen, 
Tab. 1 Ablauf des digital stattfindenden Spiegelkurses

\begin{tabular}{|l|l|l|l|}
\hline Tag & Ablauf & $\begin{array}{l}\text { Dauer } \\
\text { (min) }\end{array}$ & $\begin{array}{l}\text { Leistungs- } \\
\text { kontrolle }\end{array}$ \\
\hline 1 & $\begin{array}{l}\text { Online-Demonstration durch Oberarzt nach der Peyton- } \\
\text { Methode }\end{array}$ & 45 & - \\
\cline { 2 - 4 } & $\begin{array}{l}\text { UE 1/UE 2 } \\
\text { Üben in Zweiergruppen unter Aufsicht der Peers und } \\
\text { ärztlicher Supervision }\end{array}$ & 90 & 1 \\
\hline 2 & $\begin{array}{l}\text { UE 3 } \\
\text { Üben in Zweiergruppen unter Aufsicht der Peers und } \\
\text { ärztlicher Supervision }\end{array}$ & 45 & 2 \\
\hline 3 & $\begin{array}{l}\text { UE 4 } \\
\text { Üben in Zweiergruppen unter Aufsicht der Peers und } \\
\text { ärztlicher Supervision }\end{array}$ & 45 & 3 \\
\hline 4 & $\begin{array}{l}\text { UE 5 } \\
\text { Üben in Zweiergruppen unter Aufsicht der Peers und } \\
\text { ärztlicher Supervision }\end{array}$ & 45 & 4 \\
\hline 5 & $\begin{array}{l}\text { UE 6 } \\
\text { Üben in Zweiergruppen unter Aufsicht der Peers und } \\
\text { ärztlicher Supervision }\end{array}$ & 45 & $\begin{array}{l}5 \text { Abschluss- } \\
\text { prüfung }\end{array}$ \\
\hline UE Unterrichtseinheit & & \\
\hline
\end{tabular}

dass die Implementierung digitaler Strukturen gleichermaßen von Lehrenden als auch Lernenden begrüßt wurde und neue Vernetzungsmöglichkeiten zwischen den einzelnen Fakultäten erschlossen hat [6, 15]. Gleichwohl lag der Fokus bislang weitestgehend auf dem Erwerb theoretischer Kenntnisse in Form geeigneter E-LearningFormate. Die digitale Vermittlung praktischer Fertigkeiten erschien hingegen zu komplex.

Analog zur Präsenzlehre ist auch in der Online-Version das Prinzip des "constructive alignment" [2], d.h. der didaktischen Kohärenz, zu wahren, durch welches Lernaktivität, Lernziele und Prüfungsformate in bestmöglichen Einklang gebracht werden sollen [1, 12, 14, 17, 23]. Der Nationale Kompetenzbasierte Lernzielkatalog Medizin (NKLM) und die Approbationsordnung für Ärzte (ÄApprO) geben hierbei die Lernziele vor und stellen auch Qualitätsanforderungen an Prüfungsformate [1, 12]. Unter Beachtung dieser Vorgaben und der neuen Online-Bedingungen bleibt vorerst ungeklärt, ob eine standardisierte Vermittlung praktischer Fertigkeiten in der HNOHeilkunde möglich ist [9].

Die Online-Lehre in der universitären HNO-Heilkunde in Freiburg erfolgt auf der offiziellen Lehrplattform "ILIAS" und basiert seit 2018 auf einem eigenständigen, webbasierten Lernprogramm [7, 10]. Im Sommersemester 2020 konnten außerdem positive Erfahrungen mit der
4-Schritt-Methode nach Peyton bei der Vermittlung komplexer praktischer Fertigkeiten gemacht werden [22]. Bisher fehlen jedoch spezifische Untersuchungen zur Effektivität dieser Methode bei digitaler Anwendung im Sinne einer Lernerfolgsüberprüfung.

\section{Zielsetzung}

Mit dieser Studie soll überprüft werden, inwieweit praktische Fertigkeiten im Rahmen eines digital stattfindenden Spiegelkurses erworben werden können. Es wird erwartet, dass die Studierenden die angebotenen Untersuchungen im Verlauf der 6 Unterrichtseinheiten (UE) zunehmend besser ausführen. Zudem soll festgestellt werden, ob manche Untersuchungen online leichter zu erlernen sind als andere.

Der Studienaufbau orientiert sich an der Studie von Polk et al., die gezeigt hat, dass für eine sichere Ausführung der Untersuchungsabläufe die Wiederholung unabdingbar ist [17]. Diese Studie wurde unter regulären Unterrichtsbedingungen in Präsenz durchgeführt und liefert somit optimale Referenzwerte bei der Beantwortung der Frage, ob die Vermittlung praktischer Fertigkeiten in der digitalen Lehre mit der der Präsenzlehre Schritt halten kann.

\section{Methodik}

\section{Stichprobe und Untersuchungs- ablauf}

Die prospektive Studie wurde im Wintersemester 2020/2021 mit 146 Studierenden im Rahmen des Blockpraktikums der HNOHeilkunde durchgeführt. Die Studierenden verteilten sich auf sechs Blöcke à 24-28Personen. Im Vorfeld sollten sich die Studierenden mithilfe von Untersuchungsvideos und Tutorials, die im HNO-Lernprogramm der Klinik hinterlegt waren [7, 10], auf den digital stattfindenden Spiegelkurs vorbereiten. Diese Methode wird als "flipped classroom" $[5,18,21]$ bezeichnet und ist ein etabliertes Konzept digitaler Lehre [3].

Vor Beginn erfolgte die Einteilung der Studierenden in Zweiergruppen unter Berücksichtigung der Corona-Verordnungen des Bundes und des Landes BadenWürttemberg sowie die Ausgabe der Untersuchungsmaterialien, welche überwiegend aus Einmal-Instrumentarium bestanden. Für Studierende mit Kontakt zu Risikopatient*innen wurde die Teilnahme mit nichtstudentischen Untersuchungspartner*innen gewährt. Die gebildeten Zweiergruppen nahmen von zu Hause aus über Videokonferenz an der digitalen Lehrveranstaltung teil.

Um adäquate Gruppengrößen von maximal 10 Studierenden sicherzustellen, erfolgte eine randomisierte Einteilung in drei Gruppen (A, B und C). Von diesen wurde vor der Randomisierung eine Gruppe als sog. Prüfgruppe (PG) bestimmt, die sich jeden Tag nach der letzten UE einer formativen Prüfung unterziehen musste $(n=48)$. Die Studierenden der anderen beiden Gruppen dienten als Kontrollgruppe (KG) für die Abschlussprüfung an Tag $5(n=98)$.

Die Online-Präsenzveranstaltungen fanden eine Woche lang täglich statt. Am letzten Tag dieser Woche wurde der Spiegelkurs durch eine summative Prüfung beendet ( $\bullet$ Tab. 1).

In der ersten Sitzung an Tag 1 wurden die Untersuchungstechniken unter ärztlicher Leitung nach der Peyton-Methode online demonstriert. In einer nachfolgenden 90-minütigen Sitzung konnten die erworbenen Kenntnisse in Zweiergruppen angewendet werden. Die Betreuung der Studierenden erfolgte dabei an den ein- 


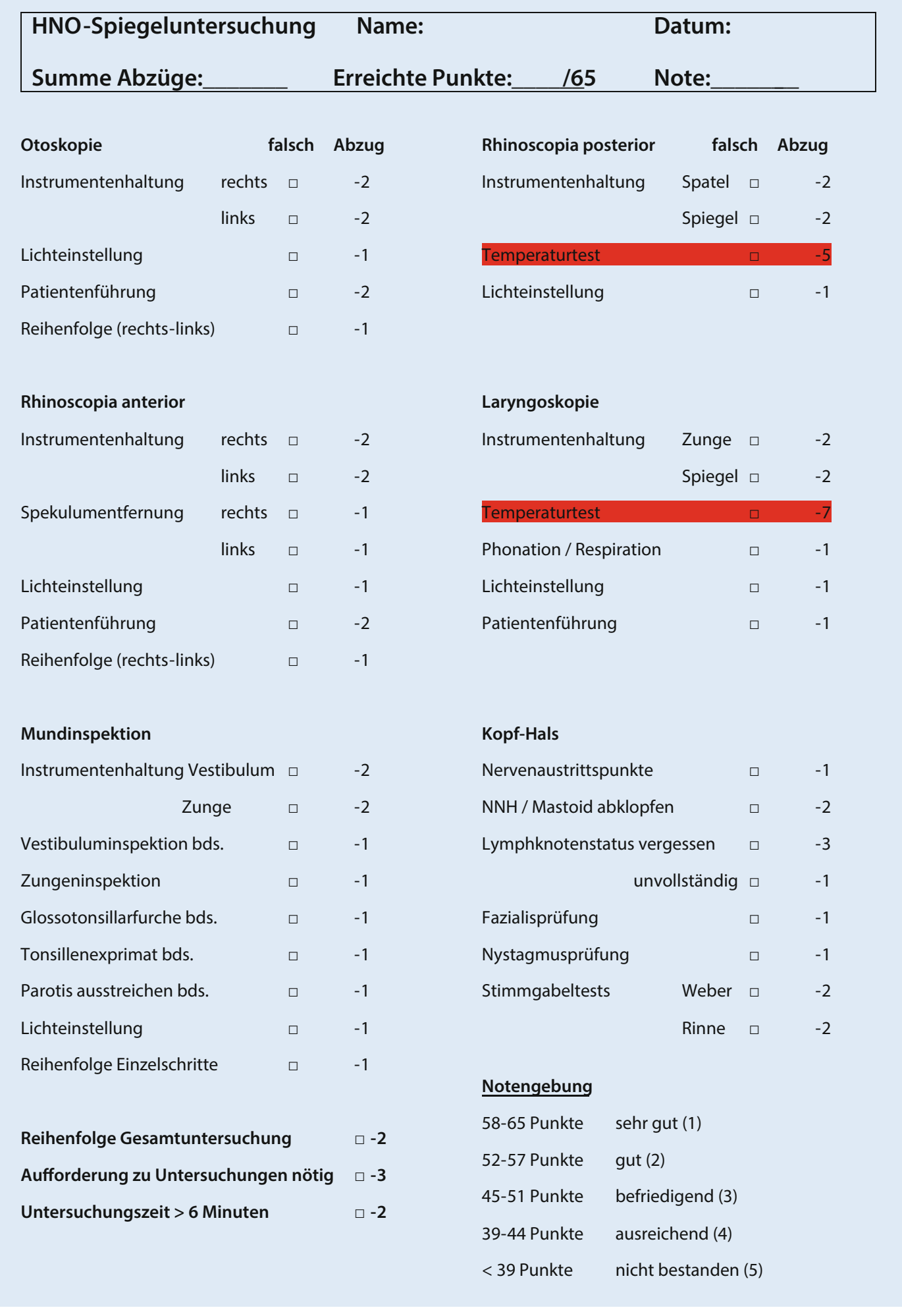

Abb. 1 Modifizierte Prüfungs-Checkliste für den HNO-Spiegelkurs. (@ Klinik und Poliklinik für HalsNasen-Ohrenheilkunde, Medizinische Fakultät Carl Gustav Carus, Technische Universität Dresden)

zelnen Tagen jeweils durch drei didaktisch und fachlich geschulte studentische Tutor*innen (sog. Peers) [8] unter ärztlicher Supervision über ein Videokonferenzsystem mit der Möglichkeit, Feedback zu den einzelnen Untersuchungstechniken zu ge- ben. Am Ende der UE 2 wurde der Lernfortschritt der Studierenden aus der PG mit einer standardisierten Checkliste ( $\bullet$ Abb. 1) erfasst, die den Studierenden nicht bekannt war [9]. Diese formative Prüfung, die nahezu identisch mit derjenigen bei
Polk et al. [17] ist, wiederholte sich an den folgenden vier Tagen. Jeweils im Anschluss erhielten die Studierenden ein Feedback der Peers. Der Prüfungsbogen umfasst die sechs Teiluntersuchungen Otoskopie, anteriore Rhinoskopie, Mundhöhleninspek- 


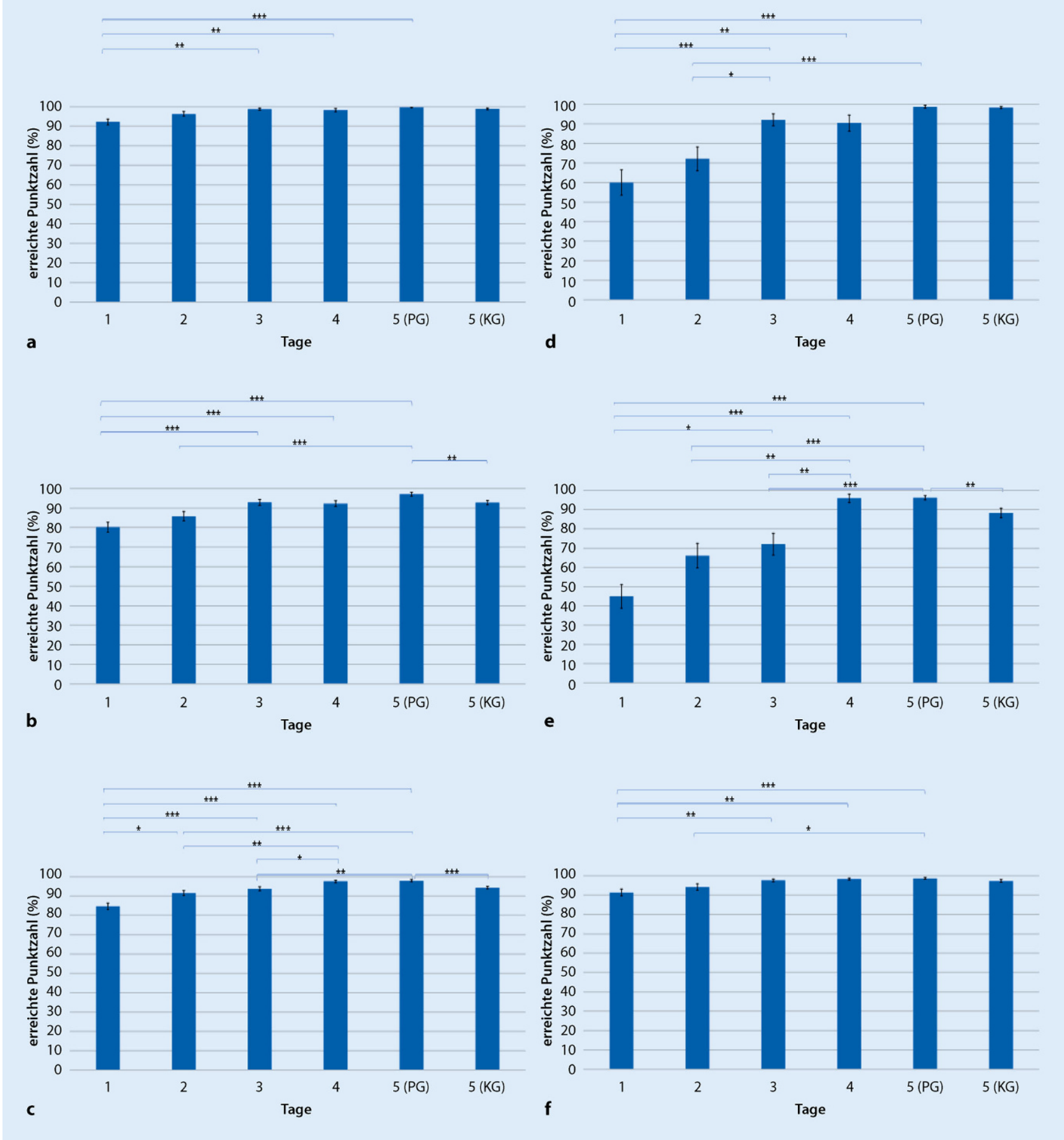

Abb. $2 \triangle$ a-f Darstellung der jeweils an den fünf Prüfungstagen prozentual erreichten Gesamtpunktzahl in den Teiluntersuchungen, ( $n_{\mathrm{PG}}=48, n_{\mathrm{KG}}=98$ ), Signifikanzniveau: ${ }^{*} \leq 0,05 ;{ }^{* *} \leq 0,01 ;{ }^{* * *} \leq 0,001$. a Otoskopie, b Rhinoscopia anterior, $\mathrm{c}$ Mundhöhleninspektion, d Rhinoscopia posterior, e Laryngoskopie, f Kopf-Hals-Untersuchung

tion, posteriore Rhinoskopie, Laryngoskopie und eine Kopf-Hals-Untersuchung, die auch in dieser Reihenfolge zu demonstrieren waren. Von maximal zu erreichenden 65 Punkten wurden bei Fehlern in Instrumentenhaltung, Lichteinstellung, Patien- tenführung und Reihenfolge für die Teiluntersuchungen jeweils Punkte abgezogen. Zusätzliche Abzüge wurden für das Überschreiten von $6 \mathrm{~min}$ Untersuchungsdauer, bei Notwendigkeit zu Aufforderungen zu einzelnen Untersuchungsschritten und bei
Nichteinhalten der Reihenfolge der Gesamtuntersuchung festgelegt. Die erreichte Note orientiert sich dabei an den von der ÄApprO definierten Prozentranggrenzen [1]. Da der Untersuchungskurs digital von zu Hause aus stattfand und die Studie- 


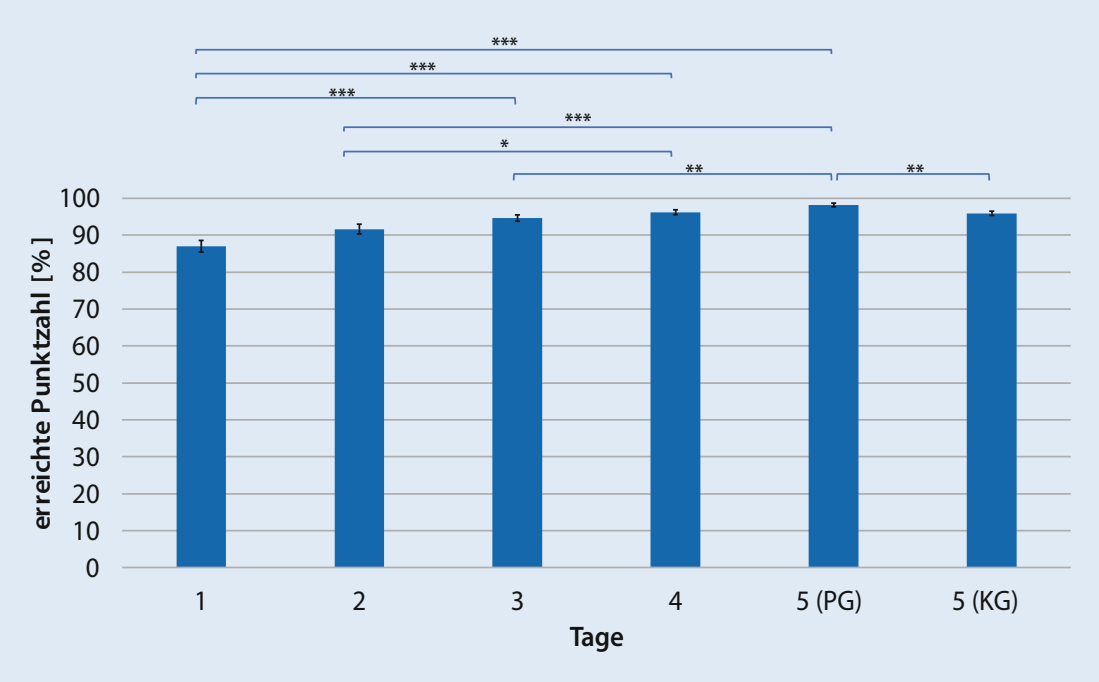

Abb. 3 \ Darstellung der jeweils an den fünf Prüfungstagen prozentual erreichten Punktzahl für die Grundfertigkeiten (Patientenführung, Lichteinstellung, Instrumentenhaltung; $n_{\mathrm{PG}}=48, n_{\mathrm{KG}}=98$ ), Signifikanzniveau: ${ }^{*} \leq 0,05 ;{ }^{* *} \leq 0,01 ;{ }^{* * *} \leq 0,001$

renden ihren Plastikspiegel lediglich mit Anti-Beschlag-Spray behandelten, war es nicht intuitiv, an einen Temperaturtest zu denken. Deshalb führte ein vergessener Temperaturtest bei den jeweiligen Teiluntersuchungen (posteriore Rhinoskopie und/oder Laryngoskopie) nicht wie bei der Studie von Polk et al. zum sofortigen Nichtbestehen, sondern es wurde lediglich die betroffene Teiluntersuchung mit 0 Punkten bewertet.

Die summative Prüfung bildete das Endergebnis des praktischen Lernzuwachses im Rahmen des Online-Spiegelkurses ab.

\section{Statistische Auswertung}

Zur Auswertung der Daten wurde SPSS 24.0 (Fa. IBM, Corp. 2015, Armonk/NY, USA) verwendet. Es wurden einfaktorielle Varianzanalysen mit Messwertwiederholung mit paarweisen Vergleichen nach Bonferroni durchgeführt. Cohen-f wurde als Maß der Effektstärke berechnet. Hierbei entspricht $f=0,10$ einem schwachen, $f=0,25$ einem mittleren und $f=0,40$ einem starken Effekt [4].

Ein Q-Test nach Cochran mit McNemarTest als Post-hoc-Analyse wurde bei der Auswertung der Untersuchungszeit eingesetzt. Das Signifikanzniveau wurde mit $p<0,05$ festgelegt.
Zum Vergleich der Ergebnisse von PG und KG bei den sechs Teiluntersuchungen und den Grundfertigkeiten im Rahmen der summativen Abschlussprüfung an Tag 5 wurde jeweils ein t-Test für unabhängige Stichproben durchgeführt und Cohen-d als Maß der Effektstärke berechnet $(d=0,2$ : kleiner, $d=0,5$ : mittlerer, $d=0,8$ : großer Effekt [4]). Für den Vergleich des Anteils an Studierenden mit eingehaltener Untersuchungszeit in PG und KG wurde ein $X^{2}$ Test eingesetzt und die Effektstärke als Cohen- $\omega$ angegeben ( $\omega=0,1$ kleiner, $\omega=0,3$ mittlerer, $\omega=0,5$ großer Effekt [4]).

\section{Ergebnisse}

\section{Teiluntersuchungen}

Bei den Teiluntersuchungen zeigten sich Unterschiede bezüglich des vorhandenen Fertigkeitsniveaus an Tag 1 (• Abb. 2a-f). Die Studierenden erzielten sowohl bei der Otoskopie als auch bei der Kopf-Hals-Untersuchung bereits am ersten Tag mehr als $90 \%$ der Punktzahl. Bei der posterioren Rhinoskopie wurde dagegen eine Punktzahl von $60 \%$ erreicht und bei der Laryngoskopie nur eine Punktzahl von 44,9\%. Darüber hinaus sind bei allen Teiluntersuchungen signifikante Unterschiede zwischen dem Ausgangsniveau an Tag 1 mit den Tagen 3, 4 und 5 festzustellen. Für den Vergleich der Ergebnisse von Tag 1 und Tag 5 wurden folgende Effektstärken (Cohen-f) ermittelt: für die Otoskopie 0,46 ; für die anteriore Rhinoskopie 0,54; für die Mundhöhleninspektion 0,73; für die posteriore Rhinoskopie 0,51; für die Laryngoskopie 0,66 und für die Kopf-Hals-Untersuchung 0,44.

Weitere signifikante Unterschiede im Fertigkeitsniveau wurden für alle Teiluntersuchungen mit Ausnahme der Otoskopie zwischen Tag 2 und Tag 5 festgestellt (- Abb. 2b-f). Zwischen dem dritten und fünften Tag konnte nur für die Mundhöhleninspektion und für die Laryngoskopie ein Leistungszuwachs ermittelt werden (• Abb. $2 c, e)$. Bei keiner Untersuchung konnte ein signifikanter Leistungszuwachs zwischen Tag 4 und Tag 5 festgestellt werden.

Analysen zum Vergleich der von der PG und der KG am fünften Tag erbrachten Leistungen zeigten nur bei der anterioren Rhinoskopie $(\mathrm{t}(137,083)=2,885 ; p \leq 0,01$; Cohen- $d=0,434)$, der Mundhöhleninspektion $\quad(\mathrm{t}(139,374)=4,117 ; \quad p \leq 0,001$; Cohen- $d=0,612$ ) und der Laryngoskopie $(t(128,901)=2,926 ; p \leq 0,01$; Cohen$\mathrm{d}=0,385)$ signifikante Unterschiede.

\section{Grundfertigkeiten (Patienten- führung, Lichteinstellung, Instrumentenhaltung)}

Wie aus $\triangle$ Abb. 3 hervorgeht, ist die von der PG erreichte Punktzahl an Tag 5 signifikant größer als an Tag 1 (Cohen- $f=0,63$ ). Weitere signifikante Punktunterschiede zeigen sich von Tag 1 auf Tag 3 und Tag 4 sowie von Tag 2 auf Tag 5 ( $p$ jeweils $\leq 0,001$ ). Auch von Tag 2 auf Tag $4(p \leq 0,05)$ und von Tag 3 auf Tag $5(p \leq 0,01)$ sind die Leistungsunterschiede signifikant. Die von der PG und der KG erreichten Punktzahlen unterschieden sich ebenfalls signifikant voneinander $(\mathrm{t}(136,083)=2,947 ; p \leq 0,01$; Cohen- $d=0,445)$.

\section{Untersuchungszeit $<6$ min}

Bei der Einhaltung der Untersuchungszeit konnte bis zu Tag 3 täglich ein signifikanter Leistungszuwachs festgestellt werden ( $p \leq 0,05$; Abb. 4). Auch verglichen mit der Anfangsleistung war bis Tag 5 eine signifikante Verbesserung in der Einhaltung der Untersuchungszeit zu beobach- 


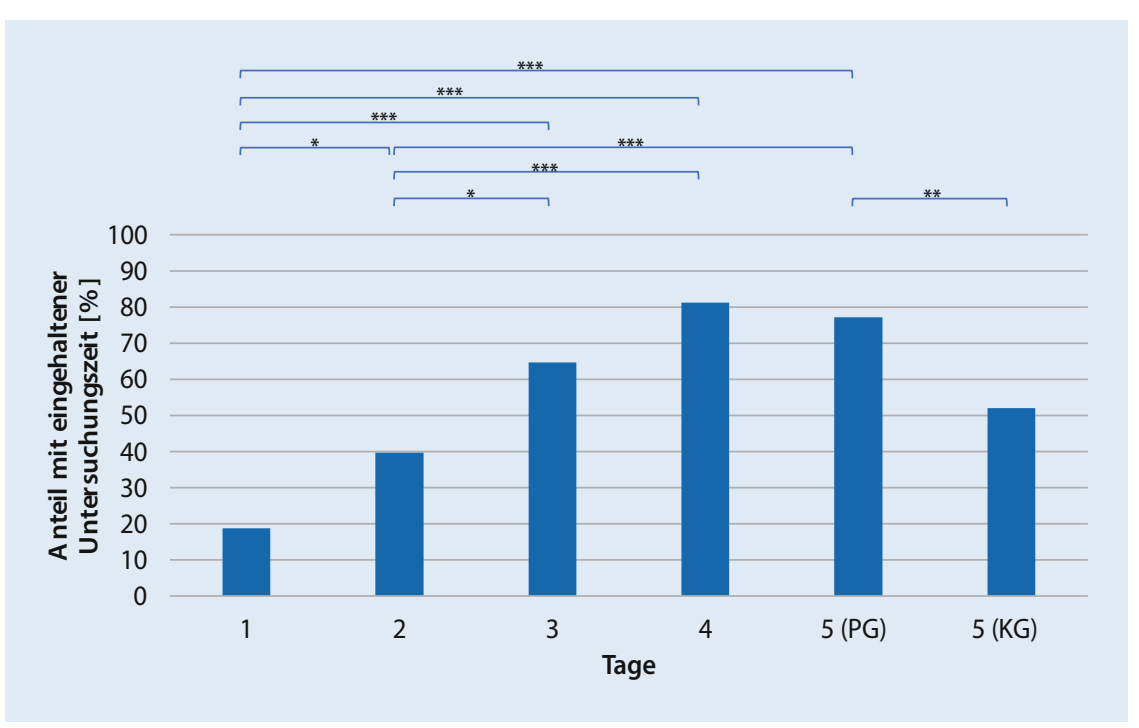

Abb. 4 A Darstellung des prozentualen Anteils der Studierenden mit eingehaltener Untersuchungszeit (<6 min) jeweils an den fünf Prüfungstagen $\left(n_{\mathrm{PG}}=48, n_{\mathrm{KG}}=98\right)$, Signifikanzniveau: ${ }^{*} \leq 0,05$; ${ }^{* *} \leq 0,01 ; * * * 0,001$

ten. Der leichte Leistungsabfall von Tag 4 auf Tag 5 war nicht signifikant. Zwischen den Leistungen der PG und der KG ist eine statistisch signifikante Differenz zu erkennen $\left(X^{2}(1, n=146)=8,439 ; p \leq 0,01\right.$; Cohen- $\omega=0,240)$.

\section{Diskussion}

Mit den Ergebnissen kann belegt werden, dass der Erwerb praktischer Fertigkeiten im Rahmen einer digital stattfindenden Lehrveranstaltung möglich ist. Für die Teiluntersuchungen des Spiegelkurses, für die Grundfertigkeiten und für die Einhaltung der Untersuchungszeit konnte ein signifikanter Lernzuwachs bei den Studierenden im Laufe der 6 UE festgestellt werden. Die ermittelten Effektstärken belegen die praktische Relevanz dieser Ergebnisse.

Mit Blick auf eine ökonomische Curriculumplanung ist aber nicht nur die Frage nach der Quantität relevant, sondern auch nach der Qualität in dem Sinne, dass ein ausgewogenes Verhältnis in puncto Lern-Zeit-Aufwand zwischen "leicht" und "schwer" zu erlernenden Fertigkeiten sichergestellt sein soll. ,"Leicht" und "schwer" sind dabei jedoch nicht dichotom zu verstehen, sondern als Kontinuum mit fließenden Übergängen. Bei der Differenzierung helfen die von der ÄApprO vorgegebenen Prozentranggrenzen und die Kompetenzebenen des NKLM $[1,12]$. Lauter et al. [11] haben in ihrer Studie Schwierigkeitsempfinden und klinische Relevanz des Erlernens verschiedener medizinischer Untersuchungen aus der Sicht von Lehrenden, Tutor*innen und Studierenden im Rahmen des Studierendenunterrichts dargestellt. Diese Einschätzungen können durch die Daten unserer Studie weitestgehend bestätigt werden. Zu den schwer zu erlernenden Fertigkeiten zählen die anteriore Rhinoskopie, die posteriore Rhinoskopie und die Laryngoskopie. Bei diesen Untersuchungen konnte erst an Tag 3 nach 4 UE, bei der Laryngoskopie sogar erst an Tag 4 nach 5 UE eine nach ÄApprO „sehr gute" Leistung erbracht werden [1]. Diese Leistungen genügen dem Anspruch der Kompetenzebene 3a („Unter Anleitung selbst durchführen und demonstrieren") des NKLM [12]. Interessanterweise definiert der NKLM auch für das Praktische Jahr (PJ) von den sechs Teiluntersuchungen nur bei der anterioren Rhinoskopie und der Laryngoskopie - die posteriore Rhinoskopie findet keine Erwähnung - die Kompetenzebene 3a als Zielwert, während es bei Otoskopie, Mundhöhleninspektion und Kopf-Hals-Untersuchung die Kompetenzebene 3b („Selbstständig und situationsadäquat in Kenntnis der Konsequenzen durchführen“) zu erreichen gilt [12]. Mit Sicherheit ist ein Grund für diese Unterscheidung darin zu sehen, dass es sich bei den als "schwer" eingestuften Fertig- keiten um genuin HNO-ärztliche Untersuchungen handelt, die für das PJ meist nur dann relevant sind, wenn das Wahlfach in der HNO-Heilkunde absolviert wird. Die als "leicht" eingestuften Fertigkeiten sind auch in anderen klinischen Fächern relevant. So spielt die Kopf-Hals-Untersuchung z. B. bei der Erhebung eines neurologischen Status eine zentrale Rolle, und sowohl die Otoskopie als auch die Mundhöhleninspektion gehören in der Pädiatrie und der Allgemeinmedizin zum Standardrepertoire. Darüber hinaus wird in Freiburg ein Famulaturreifekurs angeboten, der die Studierenden frühzeitig befähigen soll, im Rahmen der medizinischen Ausbildung aktiv an Untersuchungen teilzunehmen [20]. Hierbei werden die Otoskopie, die Mundhöhleninspektion und die Kopf-Hals-Untersuchung (•Abb. 1) nahezu vollständig abgedeckt. Unsere Studie kann dennoch verdeutlichen, dass auch bei diesen "leicht" zu erlernenden Untersuchungen über die fünf Tage statistisch signifikante Leistungssteigerungen möglich sind. Zudem zeigte sich beim Vergleich der Prüfungsergebnisse von PG und KG bei der Mundhöhleninspektion trotz guten Ausgangsniveaus ein signifikanter Unterschied (- Abb. 2c). Dies kann u.a. dadurch erklärt werden, dass einerseits eine bestimmte Untersuchungsreihenfolge der Einzelschritte einzuhalten war und andererseits manche Einzelschritte wie z.B. die Untersuchung der Glossotonsillarfurche oder die Provokation von Tonsillenexprimat ( $\mathbf{A b b}$. 1) von den Studierenden im Famulaturreifekurs noch nicht eingeübt wurde. Es ist davon auszugehen, dass sich durch die täglich stattfindenden formativen Prüfungen der PG und das anschließende Feedback eine bessere Untersuchungsroutine als bei der KG eingestellt hat.

Bei einer ressourcenoptimierten Lehrplanung ist also eine stärkere Ausrichtung auf die "schwer" zu erlernenden Untersuchungen durchaus sinnvoll, jedoch sollte auch der potenzielle Leistungszuwachs bei den "leichten" Untersuchungen maximal ausgereizt werden.

In diesem Kontext kann „maximales Ausreizen" aber auch bedeuten, dass ein Üben jenseits der Lernsättigungskurve ineffektiv ist und unnötig Ressourcen verbraucht. Bei unserer Studie konnten in allen Teiluntersuchungen an Tag 4, d. h. nach 
5 UE, über $90 \%$ der maximalen Punktzahl erreicht werden, was nach ÄApprO einer „sehr guten" Leistung entspricht [1]. Von Tag 4 auf Tag 5 trat kein signifikanter Kompetenzzuwachs mehr auf. Demnach scheint sich in der digitalen Vermittlungsversion von Tag 4 auf Tag 5 nicht die von Polk et al. postulierte "Prozessroutine" einzustellen [17]. Damit ist auch die Notwendigkeit einer sechsten UE infrage gestellt. Dennoch ist es durch die Verbesserung der Untersuchungszeit im Verlauf der fünf Tage (-Abb. 4) durchaus gerechtfertigt zu behaupten, dass sich bei der digitalen Lehre praktischer Fertigkeiten zumindest eine "Untersuchungsroutine" vollzieht. Ob diese von Dauer ist, müsste jedoch durch eine Wiederholung der formativen Prüfung mit zeitlichem Abstand zum Untersuchungskurs belegt werden.

Auf dem Weg zu dieser digital-praktisch erworbenen Untersuchungsroutine sind auch einige Störfaktoren im Gegensatz zur Präsenzlehre zu berücksichtigen, die Einfluss auf den Lernzuwachs haben können.

Ein Grundproblem war die Realisierung der ersten beiden Schritte nach der Peyton-Methode. Mittels standardisierter Kameraeinstellung in $45^{\circ}$-Position auf die untersuchte Person sollten einerseits die technische Ausführung und andererseits die erhobenen Befunde nachzuvollziehen sein. Die fehlende natürliche „Autofokussierung" führte diese Herangehensweise jedoch an ihre Grenzen. Wir begegneten dem Problem mit makroskopischer wie auch endoskopischer Demonstration der Befunde sowie in fusionierter Darstellung im Videotutorial.

Zudem stellten wir in den jeweiligen Kurssitzungen sicher, dass sich über eine Verbindung der Schritte 3 und 4 der Peyton-Methode, die dem Grundgedanken der "circulation" von Nikendei et al. als Aufhänger diente [13], alle Studierenden aktiv beteiligen konnten. Dies erfolgte durch wechselnde Anleitung von Studierenden zu Teiluntersuchungen. Die Tutor*innen gaben jeweils am Ende der Untersuchung ein Feedback. Diese Modifikation wurde für den Kleingruppenunterricht übernommen, da die originale Peyton-Methode auf eine 1:1-Betreuung angelegt ist [22].

\section{On the effectiveness of digital teaching of practical skills in curricular ENT education}

Background: Due to the current COVID-19 pandemic, the curricular courses at faculties of medicine had to be digitalized within a short time. This poses a particular challenge regarding the teaching of practical skills.

Objective: To determine to what extent practical skills can be learned within the framework of a fully digitally implemented ENT mirror examination, the minimum number of teaching units (UE) required for competent execution, and the difficulty of the various examinations were identified.

Materials and methods: During a period of 5 days of their ENT block internship, a total of 146 students learned six ENT mirror examinations in daily videoconferences. A subgroup ( $n=48$ ) was assessed formatively each day and the increase in performance was statistically analysed over the 5 days. On the fifth day, exams were carried out with the entire cohort.

Results: Over the course of the 5 days, a significant increase in performance was observed in the various examinations. Based on the evaluation of the increase in performance in the subsections, examinations could also be classified as "simple" and "difficult." Peak performance according to competence level 3a of the Nationaler Kompetenzorientierter Lernzielkatalog Medizin (NKLM) was achieved on day 4 after 5 UE had been completed.

Conclusion: Practical skills can also be acquired through digital courses. Limitations result from the quality of the examination instruments and the diagnostic findings. Additionally, economical curriculum planning can be achieved by a suitable weighting of the focus of the examination.

\section{Keywords}

COVID-19 Digitalization $\cdot$ Measuring learning outcomes $\cdot$ Blended learning $\cdot$ Medical students

Methodenkritisch ist die Qualität des Einmal-Untersuchungsinstrumentariums einzuschätzen. Die Spiegel wiesen eine vergleichsweise geringe Winkelung auf. Zudem erfolgte die Beschaffung einer Lichtquelle in studentischer Eigenverantwortung mit resultierenden Qualitätsunterschieden der Lichtverhältnisse. Die Summe beider Probleme führte v.a. bei der posterioren Rhinoskopie und der Laryngoskopie zu erschwerten Bedingungen bei der klinischen Befunderhebung. Bei Befindlichkeiten (Würgereflex) des*der Untersuchungspartner*in war ein Wechsel ausgeschlossen. Zudem sind die einzelnen Items der Checkliste (- Abb. 1) nicht näher spezifiziert. So geht z.B. aus dem Item „Instrumentenhaltung" nicht automatisch die korrekte Instrumentenhaltung hervor. Dennoch darf deren Kenntnis bei prüfenden HNO-Ärzt ${ }^{*}$ innen vorausgesetzt werden und wurde entsprechend den Studierenden in den UE vermittelt. Limitationen aufseiten der Lehrenden in Form einer empfundenen „räumlichen Barriere" fanden ihren Ausdruck in Tonund Verbindungsproblemen, fehlender natürlicher "Autofokussierung" sowie taktilen Defiziten (z.B. Korrekturen bei der Instrumentenhaltung).

Dem wäre gegenüberzustellen, dass im häuslichen Umfeld mit den Materialien ein häufigeres und zeitlich unabhängiges Üben mit dem*der Partner*in möglich war, wenngleich pandemiebedingt nicht in einem klinischen Setting.

Fazit für die Praxis

- Praktische Fertigkeiten können auch digital vermittelt werden.

- Die Peyton-Methode nimmt hierbei eine Schlüsselposition ein.

- Die digitale Ausrichtung eines klinischen Untersuchungskurses erfordert eine sorgfältige Planung sowie Strukturierung.

- Anhand der Einteilung der Teiluntersuchungen in "leicht" und "schwer" zu erlernende kann durch eine Gewichtung des Unterrichtsfokus eine ökonomische Curriculumplanung gelingen.

- Tägliche formative Prüfungen der Studierenden fördern die Ausbildung einer Untersuchungsroutine. 


\section{Korrespondenzadresse}

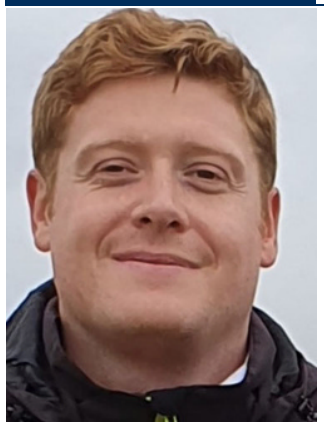

\section{F. Krauss}

Klinik für Hals-Nasen-Ohrenheilkunde, Medizinische Fakultät, Universitätsklinikum Freiburg, Albert-Ludwigs-Universität Freiburg Killianstr. 5, 79106 Freiburg, Deutschland fabian.krauss93@t-online.de

\section{Einhaltung ethischer Richtlinien}

Interessenkonflikt. F. Krauss, M. Giesler und C. Offergeld geben an, dass kein Interessenkonflikt besteht.

Alle beschriebenen Untersuchungen wurden mit Zustimmung der zuständigen Ethik-Kommission (Antragsnummer 20-1135), im Einklang mit nationalem Recht sowie gemäß der Deklaration von Helsinki von 1975 (in der aktuellen, überarbeiteten Fassung) durchgeführt. Von allen beteiligten Studierenden liegt eine Einverständniserklärung vor.

\section{Literatur}

1. Approbationsordnung für Ärzte (2002) https:// www.gesetze-im-internet.de/_appro_2002/ BJNR240500002.html. Zugegriffen am 01. Februar 2021

2. Biggs J (1996) Enhancing teaching through constructive alignment. High Educ 32(3):347-364

3. Chen F, Lui AM, Martinelli SM (2017) A systematic review of the effectiveness of flipped classrooms in medical education. Med Educ 51(6):585-597

4. Cohen J (1988) Statistical power analysis for the behavioral sciences, 2. Aufl. Lawrence Erlbaum, New Jersey

5. Dombrowski T, Dazert S, Volkenstein S (2019) Digitale Strategien in der Lehre. Laryngol Rhinol Otol 98:197-219

6. Freiherr von $\mathrm{Sa}$ P, Klenzner $\mathrm{T}$, Scheckenbach $\mathrm{K}$ et al (2017) Einsatz von E-Learning an deutschen Universitäts-HNO-Kliniken. Laryngol Rhinol Otol 96:175-179

7. http://hno-lernprogramm.uniklinik-freiburg.de. Zugegriffen: 1.Febr. 2021

8. Kemper M, Linke J, Zahnert T et al (2014) Peer Teaching und Peer Assessment sind effektive Lehrinstrumente in der HNO-Heilkunde. Laryngol Rhinol Otol 93:392-397

9. Kemper M, Zahnert T, Graupner A et al (2011) Die Operationalisierung der HNO-Spiegeluntersuchung. Laryngol Rhinol Otol 90:537-542

10. Lang F, Everad B, KnopfAetal(2020)Digitalisierung in der curricularen Lehre: Erfahrungen mit dem Freiburger HNO-Lernprogramm. Laryngol Rhinol Otol.https://doi.org/10.1055/a-1334-4274
11. Lauter J, Branchereau S, Herzog Wet al (2017) TutorielleVermittlung klinisch-praktischer Fertigkeiten im Skills-Lab:Komplexität, Relevanz und Lehrkompetenz aus ärztlicher, tutorieller und studentischer Sicht.ZEvid Fortbild Qual Gesundhwes 122:54-60

12. Medizinischer Fakultätentag der Bundesrepublik Deutschland e. V. (2015in) Nationaler Kompetenzbasierter Lernzielkatalog - NKLM. Verabschiedet auf der Mitgliederversammlung des 76. Ordentlichen Medizinischen Fakultätentages am 04.06.2015 in Kiel. http://www.nklm.de/files/ nklm_final_2015-07-03.pdf.Zugegriffen: 1. Febr. 2021

13. Nikendei C, Huber J, Stiepak J et al (2014) Modification of Peyton's four-step approach for small group teaching - a descriptive study. BMC Med Educ 14:68

14. Offergeld C, Ketterer $M$, Neudert $M$ et al (2020) „Ab morgen bitte online": Vergleich digitaler Rahmenbedingungen der curricularen Lehre an nationalen Universitäts-HNO-Kliniken in Zeiten von COVID-19. HNO 69(3):213-220

15. Offergeld C, Neudert M, Emerich M et al (2020) Vermittlung digitaler Kompetenzen in der curricularen HNO-Lehre: abwartende Haltung oder vorauseilender Gehorsam? HNO 68(4):257-262

16. Offergeld C, Praetorius M, Neudert M (2020) „ENT-expect no teaching" ...?! Welchen Stellenwert haben Aus-, Weiter- und Fortbildung in der deutschen HNO-Heilkunde? HNO 68(4):229-230

17. Polk ML, Lailach S, Kemper Met al (2020) Lernkurve der HNO-Spiegeluntersuchung: Zielgerichtete Lehrveranstaltungsplanung zu einer psychomotorischen Fertigkeit. HNO68(4):238-247

18. Prober CG, Khan S (2013) Medical education reimagined: a call to action. Acad Med 88(10):1407-1410

19. https://www.pr.uni-freiburg.de/pm/2020/ praesenzlehre-ist-unersetzlich. Zugegriffen: 1. Febr. 2021

20. Streitlein-Böhme I, Böhme K, Brich J et al (2015) Manual zur Praxis der Anamneseerhebung und körperlichen Untersuchung, Studiendekanat der Medizinischen FakultätFreiburg, 6. Aufl., Freiburg

21. Tolks D, Schäfer C, Raupach T et al (2016) Eine Einführung in die Inverted/Flipped-ClassroomMethode in der Aus- und Weiterbildung in der Medizin und den Gesundheitsberufen. GMS J Med Educ 33(3):Doc 46

22. Walker M, Peyton JWR (1998) Teaching in theatre. In: Peyton JWR (Hrsg) Teaching and learning in medical practice. Manticore Publishers Europe, Rickmansworth, S 171-180

23. Wissenschaftsrat (2020) Neustrukturierung des Medizinstudiums und Änderung der Approbationsordnung für Ärzte. Empfehlungen der Expertenkommission zum Masterplan Medizinstudium 2020. https://www.wissenschaftsrat.de/ download/archiv/7271-18. Zugegriffen: 2. Febr. 2021

\section{HNO-Veranstaltungen}

Liebe Leserinnen und Leser, Informationen zur nächsten Jahrestagung der DGHNO-KHC finden Sie hier: https://www.hno.org/2022/index.html

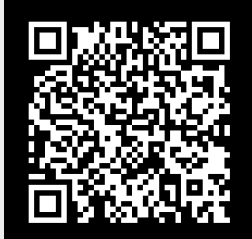

Weitere Veranstaltungen finden Sie unter: https://www.hno.org/ veranstaltungen/index.html

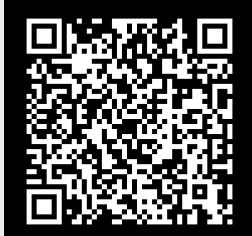

